\title{
Low Complexity Multiuser Scheduling in MIMO Broadcast Channel with Limited Feedback
}

\author{
Feng She, Hanwen Luo, and Wen Chen \\ Department of Electronic Engineering \\ Shanghai Jiaotong University \\ Shanghai 200030, P. R. China \\ Email: \{shefeng630;wenchen\}@sjtu.edu.cn
}

\begin{abstract}
This paper deals with the design and analysis of low complexity user scheduling algorithm in multi-antenna broadcast (downlink) systems under zero-forcing multiplexing with limited feedback. By using quantization technology, the channel matrix can be divided into serval unoverlapped channel regions. Based on the quantized channel regions, we can get semi-orthogonal region sets. Then the transmitter can carry out user scheduling by using the feedback channel direction information (CDI), channel quality information (CQI) and the quantized semi-orthogonal channel region sets. Simulation results show that the presented user scheduling algorithm can achieve a sum rate close to the full searching algorithms while with much lower complexity than those of the previous algorithms.
\end{abstract}

\section{INTRODUCTION}

Multiple-input multiple-output (MIMO) system is well motivated for wireless communications through fading channels due to the potential improvements in transmission rate or diversity gain [1]. It is well known that multiple antennas can be easily deployed at base station in cellular systems. However, mobile terminals usually have a small number of antennas due to the size and cost constraint. Thus, it may not be able to obtain significant capacity benefit from the multiple transmit antennas. This is true with the transmit strategy of time division multiple access (TDMA) [2]. To solve the problem, multiuser must be served simultaneously. One way to accomplish this is called dirty paper coding (DPC), which is a multiuser encoding strategy with interference presubtraction [4]. Since DPC is with high complexity, [7] presents zero forcing dirty paper coding (ZF-DPC) as a suboptimal solution, which reduces the complexity, while ZF-DPC still has a very high implementation cost due to successive encoding. As a much simple transmit strategy, zero forcing beamforming (ZFBF) techniques have been proposed for space division multiple access (SDMA) to remove the cochannel interference in MIMO downlink systems [8], [12]. Compared with DPC and ZF-DPC, ZFBF can greatly reduce the complexity while keeping the throughput region close to optimal when the number of user $K$ is large enough [3]. Then ZFBF greatly reduces the complexity while keeping the throughput region close to optimal.

In general, finding the optimal active user sets in $\mathrm{ZFBF}$ requires an exhuastive search over all users. We consider the problem of jointly multiplexing and scheduling multiple users in the wireless downlink systems. Multiuser scheduling is the problem of allocating resource (such as power and bandwidth) in order to perform desirably with respect to criteria such as throughput or performance. This problem has attracted great interest in the recent years [13], [14]. In [14], the authors propose a semi-orthogonal user scheduling algorithm to reduce interference among different data streams. In [19], a similar idea is used to develop a greedy user sets selection, which is shown to achieve the optimal asympotic sum rate. In [3], a better user sets selection scheme based on clique (full connected subgraph) graph is proposed. Because both greedy search algorithm and clique search algorithm are of high complexity, it is necessary to find an user scheduling algorithm with low complexity. In [2], a semi-orthogonal user scheduling (SUS) algorithm is proposed. However, all these schemes are based on the assumption of perfect channel state information at the transmitter (CSIT). In practical wireless communication system, transmitter can not get perfect CSI. Thus, this motivates us to consider user scheduling partial CSI via with a rate constraint feedback channel from each mobile. we also note that though SUS is of low complexity, it can not guarantee the transmitter can get the optimal user sets. This also motivates us to find a low complexity user scheduling algorithm that can get better user sets than SUS in sum-rate sense.

In this paper, we propose a low complexity user scheduling algorithm which is based on channel quantization. The underlying idea is that the multiuser channel can be modeled as a weighted graph by quantized channel with single user channel gain as node weights.

The contributions of this paper are as follows.

1) We present a new clique graph construction algorithm with low complexity for MIMO broadcast systems.

2) We propose a low complexity user scheduling algorithm with quantized semi-orthogonal channel region sets.

The paper is organized as follows. We outline the system model in section II. In section III, we introduce the transmit strategies. The proposed scheduling algorithm is presented in section IV. We analyze the complexity in section V. The simulation results are presented in section VI. Finally we conclude this paper in section VII.

Notation used in this paper are as follows: $(\cdot)^{T}$ denotes matrix transposition, $(\cdot)^{H}$ denotes matrix conjugate-transposition, and $\operatorname{tr}(\cdot)$ is trace of channel matrix, $E[\cdot]$ denotes statistical 
expectation, and $\|\cdot\|^{2}$ denotes the mean square norm of a vector.

\section{SySTEM MODEL}

\section{A. Multiuser Broadcast Channel Model}

We consider a single-cell MIMO BC system with a single base station supporting data traffic to $K$ users. The base station is with $N_{t}$ transmit antennas and each of the user terminal has single receive antenna. We assume $K \geq N_{t}$. For simplicity, we assume that all the users experience independent fading. Thus, the signal received by user $k$ is given by

$$
\boldsymbol{y}_{k}=h_{k} x+n_{k}, \quad k \in\{1, \ldots, K\},
$$

where $x \in \mathbb{C}^{N_{t} \times 1}$ is the transmit signal vector with a power constraint $\operatorname{tr}\left(E\left[x x^{H}\right]\right)=P$, and $n_{k}$ is complex Gaussian noise with unit variance per vector component, i.e., $E\left[n n^{H}\right]=I$, and $h_{k} \in \mathbb{C}^{1 \times N_{t}}$ is the multiple-input single-output (MISO) channel gain matrix to the $k$ th user.

At transmitter, we employ the ZFBF transmit strategy. In ZFBF, the scheduler first selects an active user set $S \subset$ $\{1,2, \ldots, K\}$, where the set size $|S| \leq N_{t}$. Then, the transmitter assigns different orthogonal beamforming directions to each data stream in such a way that the interference at each receiver is completely suppressed.

Denote $h_{i}, i \in\{1, \ldots,|S|\}$ as the channel to the $i$ th active user, and define $H(S)=\left[h_{1}^{T}, \ldots, h_{|S|}^{T}\right]$. Then the transmit signal is represented as

$$
\boldsymbol{x}=\sum_{i=1}^{|S|} w_{i} s_{i},
$$

where $s_{i}$ and $w_{i}$ are data symbol, beamforming vector of the $i$ th active user, respectively. Then the received signal at the $i$ th active user is given by

$$
\boldsymbol{y}_{i}=h_{i} w_{i} s_{i}+\sum_{j=1, j \neq i}^{|S|} h_{i} w_{j} s_{j}+n_{j} .
$$

From channel realization, $H=\left\{h_{1}^{T}, h_{2}^{T}, \ldots, h_{K}^{T}\right\}$, Multiuser MIMO can be represented as a node weight graph [3]. In this paper, we assume that each user has perfect CSI, and the transmitter get partial CSI by limited feedback.

\section{B. Channel direction information (CDI) feedback model}

We assume that each user has perfect knowledge of $h_{k}$ and quantizes the direction of its channel $\tilde{h}_{k}=h_{k} /\left\|h_{k}\right\|^{2}$ to a unit norm vector $\hat{h}_{k}$. The quantization vectors are chosen from a codebook of unit norm row vectors of size $N=2^{B}$, where $B$ is the number of feedback bits which denote the index of codeword in codebook.

\section{Channel quality information (CQI) feedback model}

In addition to CDI, each user feeds back its CQI $\left(g\left(h_{k}\right)\right)$ to the transmitter for user scheduling. Generally, there are two types of definition of CQI: one is channel norm $\left(g\left(h_{k}\right)=\right.$ $\left.\left\|h_{k}\right\|^{2}\right)$, and the other is $\operatorname{SINR}\left(g\left(h_{k}\right)=S I N R_{k}\right)$. As has been pointed out in [9], when take SINR as CQI model, the system can achieve both multiplexing and multiuser gains. Thus, in this paper, the CQI will be SINR rather than just channel magnitude. We also assume that the CQI is fed back directly without quantization.

\section{Multiuser Transmit Strategies}

In this section, we briefly introduce the exemplary MIMOBC transmission schemes of ZFBF. As mentioned above, multiple transmit antennas can potentially yield an $N_{t}$-fold increase in the sum capacity, where $N_{t}$ is the number of transmit antennas. [9] showed that employing ZFBF to a set of $N_{t}$ nearly orthogonal users with large channel norms is asymptotically optimal as the number of users grow large. In multiuser MISO systems, we first select a user subset $S$ to be served together, and then build the corresponding channel matrix $H(S)$, which is defined as $H(S)=\left(\left[\tilde{h}_{\pi(1)}^{T}, \ldots, \tilde{h}_{\pi(|S|)}^{T}\right]\right)$, and the beamforming vector $w_{i} \in C^{N_{t} \times 1}, i \in S$. Then the beamforming matrix $\mathcal{W}(S)$ is given by

$$
\mathcal{W}(S)=H(S)^{H}\left(H(S) H(S)^{H}\right)^{-1} .
$$

By normalizing the $i$ th column of the $W$, we can get beamforming vector $w_{\pi(i)}$ of each active user.

In ZFBF with limited feedback, CDI feedback is sufficient for determining beamforming vectors, and CQI is necessary for user selection purpose [3].

\section{Scheduling Under ZERo-Forcing MultipleXing}

In this section, we provide scheduling algorithm based on channel quantization. For finite user number of $K$, the probability of existence of an orthogonal set is zero. Thus, we consider the user sets which are "nearly" orthogonal in scheduling scheme. To be precise, we define two vectors $v_{1}$ and $v_{2}$ to be $\alpha$-orthogonal if

$$
\frac{\left|v_{1} v_{2}^{H}\right|}{\left\|v_{1}\right\|\left\|v_{2}\right\|} \leq \alpha
$$

\section{A. Channel quantization and codebook design}

The problem of channel quantization is the problem of vector (or matrix) quantization (VQ). In this paper, we attempt to divide the channel space into several unoverlapped channel regions. In other words, the space of channel matrix is divided into $N$ non-overlapped regions. For each of these regions, there is a codeword denoting the channel vector in the region, and the set of codewords is called codebook.

We consider codebook construction from fast Fourier transform matrices [5], [6]. This class of codewords in the codebook can be thought of as subset of $m$ columns of the $N \times N$ FFT matrix [21]. More precisely, the codebook consists of $m$ distinct columns chosen from an $N \times N$ FFT matrix, with index set $\mathbf{u}=\left[\begin{array}{ll}u_{1} & u_{2} \ldots u_{m}\end{array}\right]$, denoted $\mathcal{C}_{F F T}(\mathbf{u}, N)$, be the codebook of size $N$ with codewords taken to be columns of FFT matrix. These codebook is known to achieve the smallest $\mu$ for a given $N$ in very special cases. 
Using this construction, the RMS inner product magnitude is the same for all codewords. That is,

$$
\begin{aligned}
\mu_{r m s}\left(\mathcal{C}_{F F T}\right) & =\max _{j} \sqrt{\sum_{i \neq j}\left|c_{i}^{H} c_{j}\right|^{2}} \\
& =\sqrt{\sum_{i \neq k}\left|c_{i}^{H} c_{k}\right|^{2}}, \forall c_{k} \in \mathcal{C}_{F F T} .
\end{aligned}
$$

Moreover, it is important note that under the assumption of uncorrelated Gaussian channel vectors each user channel vector is equally like to be quantized to any code index. Lastly, from (??), we can see that the correlation between very two users is only a function of the magnitude of the difference of the indices in user scheduling [11], that is,

$$
\left|c_{i}^{H} c_{j}\right|=g(|i-j|)
$$

for some function $g$. Thus, these properties of FFT based codebook will be very valuable in scheduling of users with complexity constraint.

\section{B. Selecting semi-orthogonal user sets by codebook}

The main idea of our user scheduling algorithm with quantized channel is to use semi-orthogonal codewords to construct the semi-orthogonal user sets. The user scheduling algorithm is intended for systems with $K \gg N_{t}$, and for sum-rate maximization.

Before user scheduling, we firstly construct semi-orthogonal relationship among codewords in a codebook with a certain $\alpha$ constraint which is defined in (5). Because each codeword denotes a channel region, then we can consider that the semiorthogonal relationship among codewords is the same as the semi-orthogonal relationship as channel regions. Thus, from a codebook, we can construct the quantized channel region semi-orthogonal relationship, and $\alpha$ captures pair-wise semiorthogonal relation between the region when $\frac{\left|c_{i} \cdot c_{j}^{H}\right|}{\left\|c_{i}\right\| \cdot\left\|c_{j}\right\|} \leq \alpha$, where $c_{i}$ is codewords of channel region $i$. This means that the two regions $R_{i}$ and $R_{j}$ are $\alpha$-orthogonal region (note $c_{i}$ and $c_{j}$ are codewords in region $R_{i}$ and $R_{j}$ respectively). Based on the semi-orthogonal relationship of all the quantized channel region, we can further search for semi-orthogonal channel region sets which include no more than $N_{t}$ semi-orthogonal channel regions. Then the codewords in the semi-orthogonal channel region sets satisfy the following condition:

$$
\frac{\left|c_{i} c_{j}^{H}\right|}{\left\|c_{i}\right\| \cdot\left\|c_{j}\right\|} \leq \alpha, i, j \in S,|S| \leq N_{t} .
$$

Therefore, by (8), we can consider the quantized channel regions which are denoted by the codewords as a semiorthogonal channel region set. Based on the semi-orthogonal channel region sets, the semi-orthogonal user sets can be set up. In other words, the semi-orthogonal channel region sets reflects the orthogonal relationship between users. Thus, in user scheduling, we only need to consider users in the $\alpha$-orthogonal channel regions sets. Then, by (8), the user sets (or clique graph) can be easily constructed.

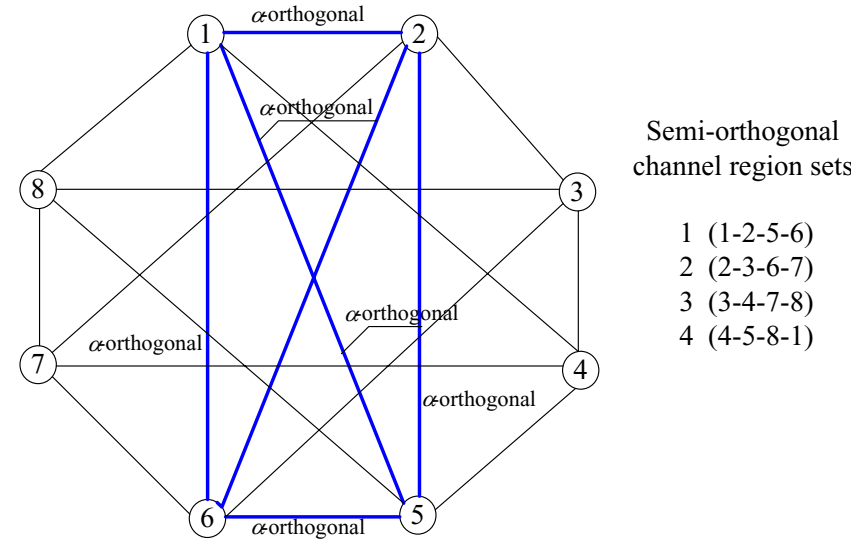

Fig. 1. The semi-orthogonal regions sets of codebook with $\alpha=0.1$ and size 8 .

With certain $\alpha$ constraint and certain codebook, we can calculate the channel region sets which meet with the semiorthogonal condition. To decide which region that the channel vectors belong to, we define distance as $d(c, h)=\left|c \cdot h^{T}\right|^{2}$. Then we use the following nearest condition

$$
\text { if } d\left(c_{i}, h\right)<d\left(c_{j}, h\right) \text { then } h \in R_{i}
$$

where $c_{i}$ and $c_{j}$ are the codewords in channel region $R_{i}$ and $R_{j}$ respectively.

Here, we express the construction of semi-orthogonal channel region sets by taking a codebook of size 8 . As mentioned above, code book of size 8 means that the channel space matrix is divided into 8 un-overlapped regions. Then as shown in Fig. 2, we can get four groups of semi-orthogonal channel region sets by $\alpha=0.1$.

In user scheduling, we only need to compare the user sets which in the corresponding semi-orthogonal channel region sets. In this way, the scheduling algorithm complexity will be decreased greatly. In the next section, we will investigate the scheduling algorithm by using the semi-orthogonal channel region sets.

\section{Low complexity scheduling algorithm with quantization}

In this section, we provide user scheduling algorithm which is based on the $\alpha$-orthogonal channel region sets. The motivation of using those sets is that the optimal $\mathrm{ZFBF}$ user sets is a clique with high probability when the number of user $K$ is very large.

\section{COMPlexity ANALYSis}

In this section, the complexity of user scheduling algorithm is analyzed. Due to DPC and greedy user selection algorithm are with high complexity, we only compare the complexity of the proposed algorithm with that of SUS algorithm which is with lower complexity than DPC and greedy user scheduling algorithm. The SUS algorithm which is mentioned in [9] consists two stages: user selection using semi-orthogonal algorithm and a beamforming weight vector calculation. We 
note that the latter stage has a small fixed complexity, requiring only one $N_{t} \times N_{t}$ matrix inversion $W(S)=H(S)^{-1}$ to obtain beamforming weights. Henceforth, we concentrate on the complexity of user selection.

In codebook based user scheduling, the main complexity lies in computing the sum rate of each semi-orthogonal group as follows

$\arg \max \sum_{k=1}^{N_{t}} \log _{2}\left(1+S I N R_{k}\right)=\arg \max \prod_{k=1}^{N_{t}}\left(1+S I N R_{k}\right)$.

Lemma 1: Assume that there are $N_{s}$ groups of semiorthogonal channel region sets under $\alpha$ constraint. Then total times of real value multiplication is $\left(N_{t}-1\right) N_{k}$, where $N_{k} \leq$ $N_{s}$ denotes the number of semi-orthogonal user sets in the time slot.

We also note that to select the highest SINR, both SUS and codebook based algorithm need to do SINR comparison among multi-users. In SUS, there need $K-1$ times of SINR comparison in the first time of iteration, where $K$ is the total number of users. Thus, SUS needs at least $K-1$ times of SINR comparison. While in the presented codebook based algorithm, the time of SINR comparison is at most $K-N_{s}$, which is much smaller than $K-1$. Thus, when $K$ is large enough, the complexity of the proposed algorithm will be much lower than that of SUS algorithm.

\section{Simulation Result And Discussion}

In this section, we provide some numerical examples to illustrate the performance of the proposed user scheduling algorithm. In the considered multiuser MIMO downlink systems, the number of transmit antenna is $N_{t}=4$, and each user has single receive antenna. Let the power in simulation be $P=10 \mathrm{~dB}$. With spatial multiplexing, the number of the active users in one time slot can not exceed the number of the transmit antennas.

We assume that the discrete-time channel impulse response is generated according to the Hiperlan2 Channel Model C in [20]. The channels between different transmit and receive antennas are assumed to be independent.

A. Experiment 1: The first experiment tests the semiorthogonal channel region sets for different $\alpha$. The codebook used in this experiment is designed by the principle in section III. Here, $N=8,16,32,64$ denote the size of the different codebooks. From Fig. 2, we can see that the number of semiorthogonal channel region sets will increase with the increment of $\alpha$ by the definition in (5). We can also find that codebooks with bigger size will have more channel region sets than that of codebooks with smaller size. This implies that big size codebook will result in higher complexity in user scheduling while get better performance. Thus, there exists a tradeoff between codebook size and performance.

B. Experiment 2: The second experiment is about the capacity of the presented user scheduling algorithm, random beamforming (RB) and semi-orthogonal user scheduling (SUS) algorithm presented in [9]. The principle of RB was

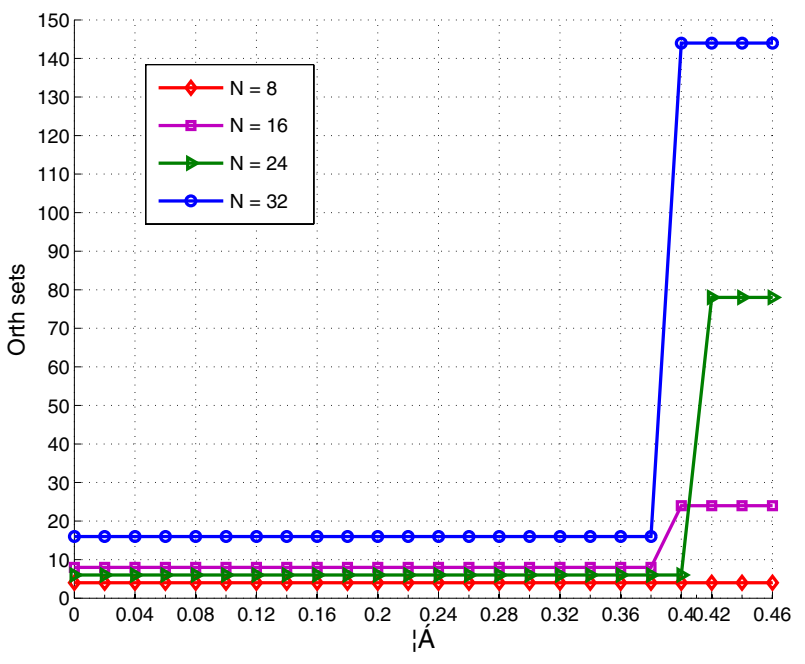

Fig. 2. The number of orthogonal sets with different codebooks.

introduced in [10]. In Fig. 3, RB1 denotes each terminal only feedback the best SINR, and RB2 and RB3 denote each user feedback the best 2 and best 3 SINR respectively. For SUS algorithm, we choose optimal $\alpha$ range from 0.25 to 0.36 . Fig. 3 shows that the presented algorithm and SUS can achieve higher capacity than that of RB. When RB is employed, more feedback information will improve system performance.Thus, there exists a tradeoff between performance and feedback loading. We can also find that when using FFT codebook of size 64 and 128, the system will achieve higher sum-rate than SUS with codebook of size 256 . While when using FFT codebook of size 32, the sum-rate is moderate lower than SUS. The result implies that the presented scheduling algorithm will achieve higher sum-rate than SUS by correctly selecting the FFT codebook size.

C. Experiment 3: The third experiment is about the complexity of the the presented scheduling algorithm and that of SUS algorithm. In this experiment, let $N_{t}=4, \alpha=0.3$, the FFT codebook size be 128 and 256 respectively, and the codebook used in SUS be of size 256. From Fig. 6, we can see that the proposed algorithm is with much lower complexity than that of SUS. This implies that the codebook based user schduling complexity is not only with much lower complexity, but also equire less amount of feedback bits than SUS algorithm .

\section{CONCLUSION}

In this paper, we present a low complexity user scheduling algorithm with channel quantization in MIMO broadcast systems with limited feedback. The objective of the user scheduling is to reduce the computing complexity of user selection and achieve sum-rate optimization. The proposed user scheduling algorithm is with very low complexity in user scheduling than previous works. We also show that the sum-capacity will increase with the increment of the number of user. This is because that with large number of users, the transmitter can 


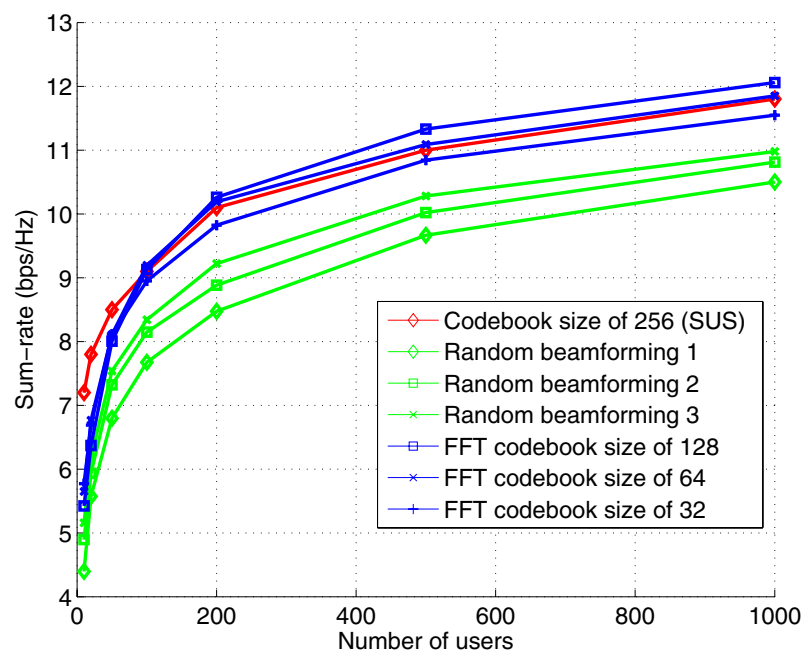

Fig. 3. The throughput of codebook based user scheduling algorithm ,random beamforming and SUS.

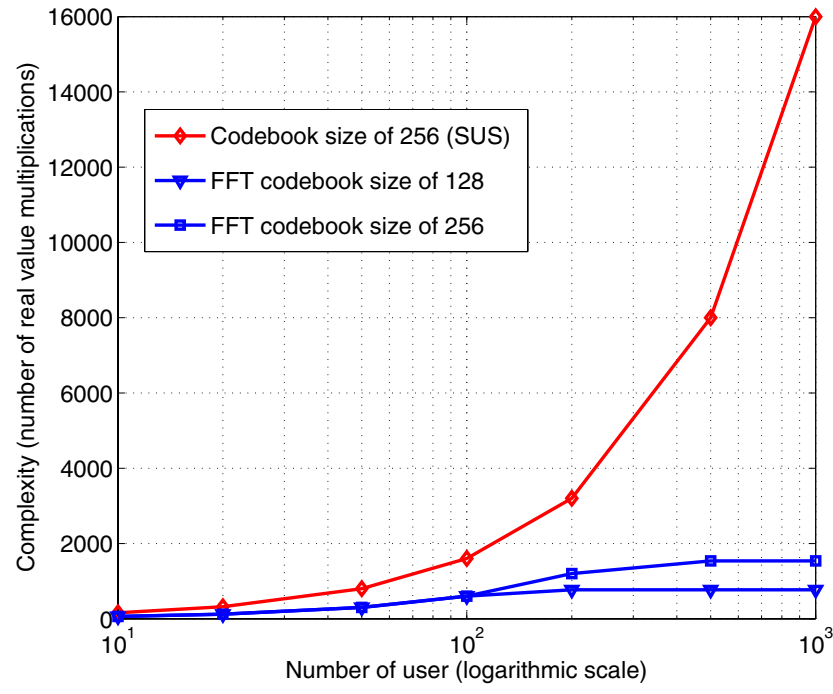

Fig. 4. The complexity of codebook based user scheduling algorithm and SUS.

choose users with good channel condition including channel gain and orthogonality among user channel vectors. In this paper, we only investigated users with single receive antenna. The case of users with multiple receive antennas would be an important extension of the paper. Moreover, how to reduce the number of feedback bits is also a very interesting problem, which will be a new practical problem.

\section{ACKNOWLEDGMENT}

The authors would like to thank Chao-Kai Xu and HongXing Li for their helpful discussion. This work is supported by NSF China \#60572157, \#60672067, by NSF Shanghai \#06ZR14041, by Shanghai-Canada NRC \#06SN07112, by
Cultivation Fund of the Key Scientific and Technical Innovation Project, Ministry of Education of China \#706022, by Program for New Century Excellent Talents in University \#NCET-06-0386, and by PUJIANG Talents \#07PJ4046.

\section{REFERENCES}

[1] S. M. Alamouti, "A simple diversity techniques for wireless communications," IEEE J. Select. Areas commun., vol. 16, pp.1451-1458, Oct. 1998.

[2] T. Yoo, A. Goldsmith, "On the optimality of multi-antenna broadcast scheduling using zero-forcing beamforming," IEEE J. Sel. Areas Commun., vol. 24, no. 3, pp. 528-541. May. 2006.

[3] T. Yoo, A. Goldsmith, "Sum-rate optimal multi-antenna downlink beamforming strategy based on clique search," in Proc. IEEE Globecom, Nov. 2005.

[4] M. H. M. Costa, "Writing on dirty paper," IEEE trans. Inf.Theory, vol. 29, no. 3, pp. 439-441. Mar. 1983.

[5] P. Xia, S. Zhou, and G. Giannakis "Achieving the welch bound with difference sets," IEEE trans. Inf. Theory. vol. 51, no. 5, pp. 1900-1907. May. 2005.

[6] C. Swannack, G. W. Wornell, and E. Uysal-Biyikoglu "MIMO broadcast scheduling with quantized channel state information" in Proc. IEEE international symposim on information theory,, Seattle, washington, Jul. 2006.

[7] G. Caire, S. Shamai, "On the achievable throughput of a multiantenna Gaussian broadcast channel," IEEE trans. Inf. Theory., vol. 49, no. 7, pp. 1691-1706. Jul. 2003.

[8] Q. H. Spencer, A.L. Swindlehurst and M. Haardt, "Zero-forcing methods for downlink spatial multiplexing in multiuser MIMO channels," IEEE Trans. Signal Processing, vol. 52, no.2 pp.461-471, Oct. 2004.

[9] T. Yoo, N. Jindal and A. Goldsmith, "Multi-antenna downlink channels with limited feedback and user selection," IEEE J. Sel. Areas Commun., vol. 25, no. 7, pp. 1478-1491. Sep. 2007.

[10] M. Sharif, B. Hassibi, "on the capacity of MIMO broadcast channels with partial side information," IEEE Trans. Inf. Theory., vol. 51, no. 2, pp. 506-522. Feb. 2005.

[11] C. Swannack, G. W. Wornell and E. U. Biyikoglu, "Efficient Quantization for Feedback in MIMO Broadcasting Systems,"in Proc. Asilomar Conference on Signals, Systems and Computers, (Pacific Grove, CA), Oct. 2006.

[12] Q. H. Spencer, C.B. Peel, A.L. Swindlehurst and M. Haardt, "An introduction to the multi-user MIMO downlink," IEEE Communications Magazine, vol. 42, no.10 pp.60-67, Oct. 2004.

[13] M. Andrews, K. Kumaran, K. Ramannan, A. Stolyar, and P. Whiting, "Prociding quality of service over a shared wireless link," IEEE Coтmunications Magazine, vol. 39, no.2 pp.150-154, Feb. 2001.

[14] P. Viswanath, D. Tse, and R. Laroia, "Opportunistic beamforming using dumb antennas," IEEE trans. Inf.Theory, vol. 48, no. 6, pp. 1277-1294. May. 2002.

[15] N. Jindal, S. Vishwanath, A. Goldsmith, "On the duality of Gaussian multiple-access and broadcast channels," IEEE trans. Inf.Theory, vol. 50, no. 5, pp. 768-783. May. 2004.

[16] H. Viswanathan, S. Venketesan, H. Huang, "Downlink capacity evaluation of cellular networks with known interference cancellation," IEEE J. Sel. Areas Commun., vol. 21, no. 5, pp. 802-811. May. 2005.

[17] W. Yu and J. M. Cioffi, "Sum capacity of Gaussian vector broadcast channels," IEEE trans. Inf.Theory, vol. 50, no. 9, pp. 1875-1892. Sep. 2004.

[18] H. Weingarten, Y. Steinberg, and S. Shamai, "The capacity region of the Gaussian multiple-input multiple-output broadcast channel," IEEE trans. Inf.Theory, vol. 52, no. 9, pp. 3936-3964. Sep. 2006.

[19] G. Dimić, N. D. Sidiropoulos, "On the downlink beamforming with Greedy user selection: Performance analysis and a simple new algorithm," IEEE trans. Signal Processing, vol. 53, no. 10, pp. 3857-3868. Oct. 2005.

[20] J. Medbo and P. Schramm, "Channel models for HIPERLAN/2 in different indoors scenarios," in ETSI/BRAN 3ERI085B, Mar. 1998.

[21] P. Xia, S. Zhou, and G. Giannakis, "Achieving the welch bound with difference sets," IEEE trans. Inf.Theory, vol. 51, no. 5, pp. 1900-1907. May. 2005. 